\title{
Early Analysis of Ambient Systems SysML Properties using OMEGA2-IFx
}

\author{
Manzoor Ahmad ${ }^{1}$, Iulia Dragomir ${ }^{1}$, Jean-Michel Bruel ${ }^{1}$, Iulian Ober $^{1}$ and Nicolas Belloir ${ }^{2}$ \\ ${ }^{1}$ IRIT, Université de Toulouse, France \\ ${ }^{2}$ LIUPPA, Université de Pau et des Pays de l'Adour, France \\ \{ahmad,dragomir,bruel,iulian.ober\}@irit.fr, belloir@univ-pau.fr
}

\author{
Keywords: \\ Requirements, Formal Verification, Observers, RELAX, Simulation
}

\begin{abstract}
:
Formal methods provide tools to verify the consistency and correctness of a specification with respect to the desired properties of the system. This verification is important as the development of an AAL (Ambient Assisted Living) system involves different technologies (medical services, surveillance cameras, intelligent devices, etc.) requiring a strong consistency checking between models. We illustrate in this paper how we prove some of the properties of the system before the development even starts. To model the AAL system, we use the SYsML language. In terms of tools, we used Rational Rhapsody in combination with the OMEGA2 profile which is an executable UML/SYsML profile used for the formal specification and validation of critical real-time systems. This profile is supported by the IFx toolset which provides mechanisms for the model simulation and properties verification of the AAL system.
\end{abstract}

\section{INTRODUCTION}

Ambient Assisted Living (AAL) systems are dedicated to help people with limited capabilities in their life. This kind of systems, and more specialy all Ambient Systems are highly adaptive. They modify their behavior at run-time in response to changing environmental conditions. For these systems, Non Functional Requirements (NFRs) play an important role, and one has to identify as early as possible the requirements that are adaptable. The distributed nature of these systems and changing environmental factors makes it difficult to anticipate all the explicit states in which the system will be during its lifetime. As such, they needs to be able to tolerate a range of environmental conditions and contexts, but the exact nature of these contexts remains imperfectly understood.

In this context, we focus on two types of system properties for our AAL case study: RELAXed and Invariant. These requirements are obtained by applying a process called RELAX [6] on traditional requirements. RELAX is a requirement engineering language for self-adaptive sys- tems that incorporates uncertainty into the specification of these systems. Our objective is to prove these properties earlier as possible in the specification cycle.

By other hand, formal methods are intended to systemize and introduce rigor into all the phases of software development. This helps us to avoid overlooking critical issues, provides a standard means to record various assumptions and decisions, and forms a basis for consistency among related activities. By providing precise and unambiguous description mechanisms, formal methods facilitate the understanding required to coalesce the various phases of software development into a successful endeavor [1].

In this paper we asses the use of formal methods for the modeling and verification of Ambient Assisted Living (AAL) systems. We use the OMEGA2-IFx approach which has been applied for the verification and validation of industrygrade models [2] providing interesting results. The OMEGA2 UmL/SysML Profile [3] defines the semantics of UML/SYSML elements providing the means to model coherent and unambiguous system models. In order to make the mod- 
els verifiable, it presents as extension the $o b$ server mechanism for specifying dynamic properties of models. The OMEGA2 UML/SysML Profile is implemented by the IFx Toolbox [4] which provides static analysis, simulation and timedautomata based model checking [5] techniques for validation.

This paper takes the reader a step forward and enriches our existing work by integrating formal methods for the verification of NFRs and specially RELAX-ed requirements.

Paper structure. This paper is organized as follows: Section 2 presents other approaches defined for the verification of AAL models, Section 3 introduces the OMEGA2 UML/SYSML Profile and the IFx Toolbox that are used for modeling and properties verification, Section 4 describes the case study: its specification, system architecture and behavior, Section 5 presents the properties, the AAL system has to satisfy, while Section 6 contains the verification and simulation results, Section 7 concludes the paper and highlights the future work.

\section{Related work}

The specification and verification of NFRs in the early stages of the AAL development cycle is a crucial issue [9]. These systems require clear and precise specifications in order to describe the system behavior and its environment. The formal specification of the system behavior supported by mathematical analysis and reasoning techniques improve their development process and enable the verification of these systems.

In [10], the authors present a verification approach based on MEDISTAM-RT which is a methodological framework for the design and analysis of real-time systems and timed traces to check the fulfillment of non-functional requirements. They focus on safety and timeliness properties, to assure the correct functioning of AAL systems and to show the applicability of this methodology in the context of this kind of systems.

In [11], the authors introduce a profile named TURTLE (Timed UML and RT-LOTOS Environment). With its formal semantics given in RTLOTOS and its toolkit, TURTLE enables a priori detection of design errors through a combination of simulation and verification/validation techniques. By simulation the authors mean a partial exploration of the system state space. It is often used for debugging purposes and to quickly increase confidence in a design. For finite state space systems, exhaustive analysis is also possible. Verification relies on the exploration of the whole system state space in order to prove absence of deadlocks for instance and other general properties that should be satisfied by any system.

In [12], the authors introduce UPPAAL which is a tool box for validation (via graphical simulation) and verification (via automatic modelchecking) of real-time systems. It consists of two main parts: a graphical user interface and a model-checker engine. The idea is to model a system using timed automata, simulate it and then verify properties on it. Timed automata are finite state machines with time (clocks). A system consists of a network of processes that are composed of locations. Transitions between these locations define how the system behaves. The simulation step consists of running the system interactively to check that it works as intended. Then the verifier check the reachability properties, i.e., if a certain state is reachable or not.

\section{THE VERIFICATION TOOLS}

In this section, we introduce the OMEGA2 Profile which we used for modeling the AAL system and its properties and the IFx toolset used for the verification and simulation of these properties.

\subsection{The OMEGA2 UML/SysML Profile}

OMEGA2 is an executable UML/SysML profile dedicated to the formal specification and validation of critical real-time systems. It is based on a subset of UML 2.2/SysML 1.1 containing the main constructs for modeling system structure and class/block behavior and which provides a clear and coherent operational and timed semantics.

The architecture of an OMEGA2 model is described in Class/Block Definition Diagrams by classes/blocks with their relationships (association, generalization and composition) and their interfaces. Each class/block defines properties and operations, as well as a state machine. The hierarchical structure of a model is defined in composite structures/Internal Block Diagrams: parts that communicate through ports and connectors. The UML/SysML Profile leaves 
open several semantic variation points for which OMEGA2 defines a set of well-formedness rules that result in a strong typing language. For further details on the rules, their rationale and formalization, the reader is referred to [13].

The behavior of a system is given by the modeled state machines that use asynchronous operation calls and signal outputs for communication. The profile owns a textual action language compatible with UML 2.2 action metamodel from which implements the main constructs: object creation/destruction, expression evaluation, variable assignment, signal output and control flow structuring statements.

The operational semantics of OMEGA2 relies on an asynchronous timed execution model. Each class/block is represented by a timed input/output automata, potentially executing in parallel with other blocks and communicating via asynchronous operation calls and signals.

The OMEGA2 Profile can model timed behavior, where the model time base can either be discrete or continuous and it is specified by the user at verification. The time model is controlled by primitives from automata with urgency [14]: clocks, time guards and transition urgency annotations. The clock is represented by a Timer block on which we can perform actions as: set for setting the clock a delay and reset to restore the clock to 0 . Time guards are either described as inequalities or specified via the timeout operation that verifies that a certain delay has elapsed. With respect to time progress, transitions can also define a particular semantics based on their stereotype: eager defines that time progress is disabled in a state (i.e., the actions on a transition are executed as soon as possible), delayable means that the time progress is enabled but it is bounded by a limit and lazy specifies that time progress is enabled and unbounded (i.e. time can progress to infinity). Based on these notions, one can also model synchronous communication in OMEGA2 model.

For specifying and verifying dynamic properties of models, OMEGA2 uses the notion of observers. Observers are special classes/blocks monitoring run-time state and events. They are defined by classes/blocks stereotyped with $<<$ observer >>. They may have local memory (attributes) and a state machine describes their behavior. States are classified as $\langle<$ success $\rangle>$ and $<<$ error >> states to express the (non)satisfaction of safety properties. The main issue in modeling observers is the choice of events which trigger their transitions, and which must include specific UML/SysML event types. One can observe:

- Events related to signal exchange: send, receivesignal, acceptsignal.

- Events related to operation calls: invoke, receive (reception of call), accept (start of actual processing of call - may be different from receive), invokereturn (sending of a return value), receivereturn (reception of the return value), acceptreturn (actual consumption of the return value).

- Informal events explicitly specified by the modeller using the informal action.

The trigger of an observer transition is a match clause specifying the type of event (e.g., receive), some related information (e.g., the operation name) and observer variables that may receive related information (e.g., variables receiving the values of operation call parameters). Besides events, an observer may access any part of the state of the UML model: object attributes and state, signal queues.

\section{$3.2 \quad$ IFx Toolset}

OMEGA2 models can be simulated and properties can be verified using the IFx toolset [15]. The following terminology is used:

Verification: It designates the automatic process of verifying whether an OMEGA2 UML/SysML model satisfies (some of) the properties (i.e. observers) defined on it. The verification method employed in IFx is based on systematic exploration of the system state space (i.e., enumerative model checking).

Simulation: It designates the interactive execution of an OMEGA2 UML/SYSML model. The execution can be performed step-by-step, random, or guided by a simulation scenario (for example an error scenario generated during a verification activity).

The IFx toolset relies on a translation of UML/SYSML models towards a simple specification language based on an asynchronous composition of extended timed automata, the IF language $^{1}$. The translation takes an input model in XMI 2.0 format. The compiler verifies the set of well-formedness rules imposed by the profile and generates an IF model that can be further reduced by static analysis techniques. This model is subject to verification that either validates the model

\footnotetext{
${ }^{1}$ http://www-if.imag.fr/
} 
with respect to its properties or produces a list of error scenarios that can be further debugged using the simulator. The overall workflow of IFx Toolset is shown in Fig. 1 [16].

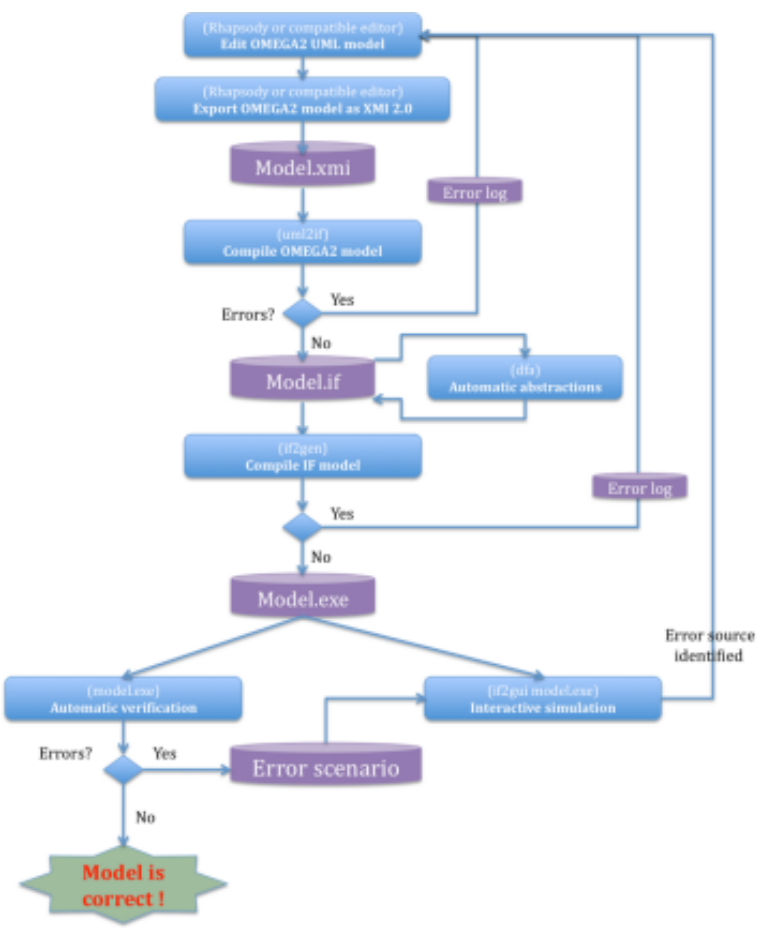

Figure 1: IFx Workflow

\section{MODELING THE AAL SYSTEM WITH OMEGA2 PROFILE}

In this section, we explore the AAL system specification, the system architecture showing its structural and behavioral diagrams i.e. its block definition diagram, internal block diagrams and state machine diagrams.

\subsection{System Specification}

Here is an excerpt of the AAL case study [6]: Mary is a widow. She is 65 years old, overweight and has high blood pressure and cholesterol levels. Following her doctors instructions, she is considering to loose weight. The doctor has recommended a hypo caloric diet with low levels of salt. She lives by herself in an AAL house. First, we start by taking into account the structural part of the AAL system. We consider those parts that are concerned with the daily calories intake of the Patient in the AAL house. The AAL system is composed of Fridge and Patient. We would like to model these parts and the interaction that takes place between them. The Fridge partially contributes to the minimum liquid intake of the Patient; it also looks at the calories consumption of the Patient as he/she does not need to exceed it after a certain threshold.

\subsection{System Architecture}

Fig. 2 shows the main internal block diagram. The important parts of the AAL system are Patient and Fridge. A Fridge in turn is composed of Food, Display, Alarm and Controller blocks. The Food block contains information about the food items in the Fridge, the calories contained by each item, the total number of calories the Patient has accumulated and the calories threshold that should not be surpassed. The fridge Display is used to show the amount of calories consumed by the Patient. The Alarm is activated in case the Patient's calories level surpasses a certain threshold. The Controller transfer the information from the Patient to the concerning elements and back to the environment.

The communication between different blocks takes place through ports. A port bears a type. In OMEGA2, the type of a port must be an Interface. The type specifies the set of requests (operation calls and/or signals) that are transferred between parts (components) by means of ports and connectors. In Fig. 2, the Patient block has a standard port named pToFridge. This port has a contract named Patient2Fridge and is acting as a provided interface of the Patient block. The interface Patient2Fridge defines an operation eat (int item, int quantity). This interface is then used as a type of pToFridge port. At the same time the Patient block has a required interface named pFromPatient. For the full system architecture, the reader is referred to [17].

Rational Rhapsody Developer v7.5.2 $2^{2}$ is used to create OMEGA2 models. OMEGA2 models use a profile and a predefined library provided with the tool (OMEGA2.sbs and OMEGA2Predefined.sbs). Any other UML2.2 or SYSML1.1 editor supporting profiling and exporting in the XMI2.0 standard compatible with Eclipse ecore can be used for OMEGA2 models.

\footnotetext{
${ }^{2}$ http://www.ibm.com/developerworks/rational/
} 


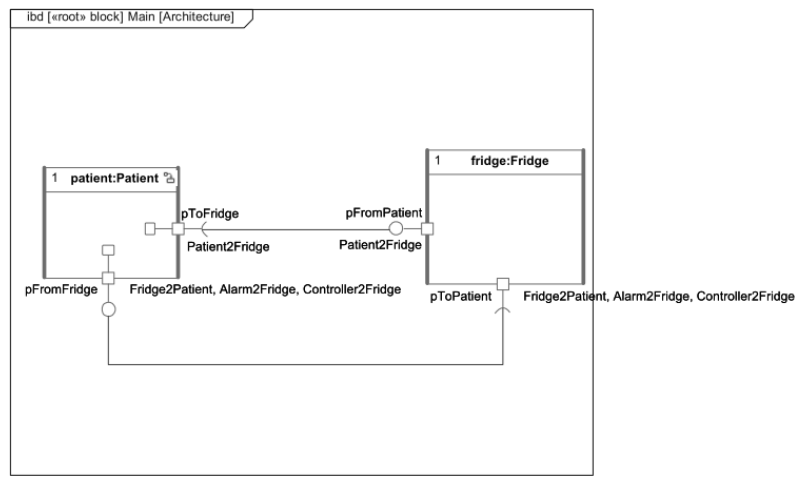

Figure 2: Main Internal Block Diagram

The Fridge interacts with the AAL system. Fig. 3 shows the internal block diagram for the Fridge block. Each of the four blocks behaviors is modeled in a separate state machine diagram.

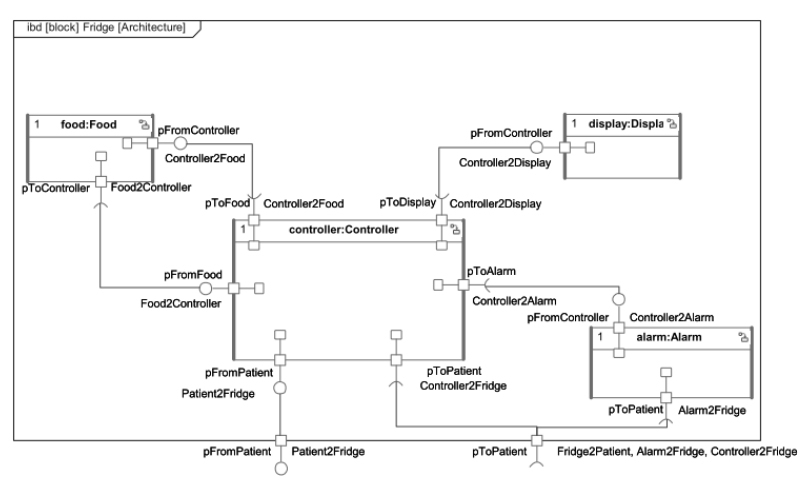

Figure 3: Fridge Internal Block Diagram

Fig. 4 shows the state machine diagram for the Patient block. Here the exchange of information between Patient and Fridge takes place. We identify the number and quantity of each item present in the Fridge. If a certain product still present in the Fridge is chosen by the Patient then the information is communicated with the Fridge. Otherwise the Fridge is empty and the Patient will wait to be refilled. Also, if the Alarm of the Fridge is raised due to high intake of calories, the Patient stops eating and waits for the system to be unblocked.

The Food block models the knowledge of the Fridge about what it contains. We define the number of items and the amount of calories associated with each item present in the Fridge. We then calculate the total number of calories accumulated by the Patient. If the total number of calories is greater or equal to the maximum calories allowed for the Patient, then a message is sent and the alarm is raised or if the total number of calories is greater than the maximum calories

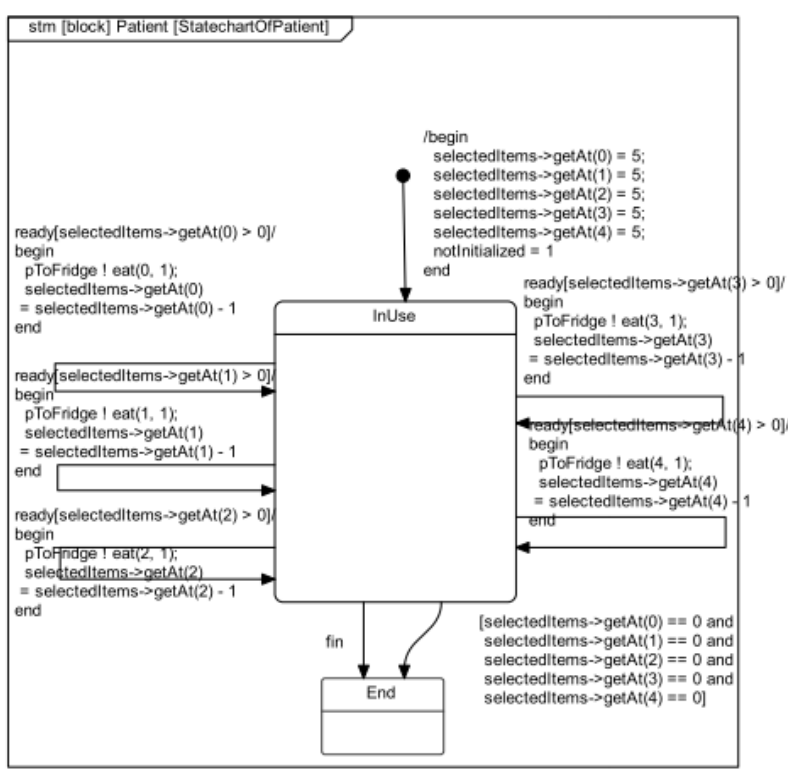

Figure 4: Patient State Machine Diagram

allowed minus 500, then the Patient is warned with a message that the calories level is approaching the maximum amount of calories allowed.

\section{PROPERTIES VERIFICATION OF AAL SYSTEM}

The properties of AAL system that we modeled and verified are obtained after RELAX process is applied on its traditional requirements. RELAX is a requirement engineering language for selfadaptive systems that incorporates uncertainty into the specification of these systems. Typical textual requirements prescribe behavior using a modal verb such as SHALL that defines functionality that a software system must always provide. For self adaptive systems such as AAL, however, environmental uncertainty may mean that it is not always possible to achieve all of those SHALL statements; or behavioral uncertainty may allow for trade-offs between SHALL statements to RELAX non-critical statements in favor of other, more critical ones. Therefore, RELAX identifies two types of requirements: one that can be RELAX-ed in favor of other ones called variant or RELAX-ed and other that should never change called invariant. Below are the properties to be verified. 


\subsection{Traditional/Relax-ed Requirement}

We have studied two RELAX-ed requirements:

- The fridge shall detect and communicate with food packages

RELAX-ed version of this requirement is as follows:

- Property 1 : The fridge SHALL detect and communicate information with AS MANY food packages AS POSSIBLE

Below are the uncertainty factors associated with the given RELAX-ed requirement.

ENV: Food locations, foot item information (type, calories), food state (spoiled and unspoiled)

MON: RFID readers, Cameras, Weight sensors

REL: RFID tags provide food locations and food information; Cameras provide food locations (Cameras provide images that can be analyzed to estimate food locations), Weight sensors provide food information (whether eaten or not)

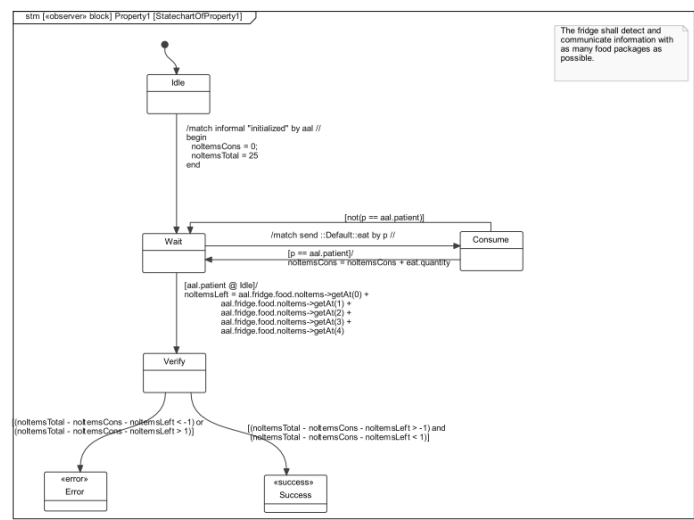

Figure 5: Property1 State Machine Diagram

The satisfaction of this requirement contributes to the balanced diet of the Patient. We would like to verify this property as it is important for the AAL system to know about as many food items as possible present in the Fridge. Fig. 5 shows the state machine diagram of the Property 1. In this property, we identify the number of items consumed by the Patient and the total number of items in the Fridge. First of all, we verify the identity of the Patient, if the person is identified as the Patient, then we calculate the number of items consumed. We then calculate the number of items left in the Fridge which is equal to the sum of all the items present in the Fridge. Then in the last step, we calculate if ((total number of items - number of items consumed - number of items left) $>-1)$ and ((total number of items - number of items consumed - number of items left) $<1$ ), it means that we have reached the <<success > state by having information about all the items present in the Fridge, i.e. it should be 0 (which means that there is no information loss). Inversely, if it is less than -1 and greater than 1 , then it means that we are missing information about some of the items present in the Fridge and the observer passes into the <<error >> state.

\subsection{Invariant Requirement}

We have taken two examples of invariants:

- Property 2 : The alarm SHALL be raised instantaneously if the total number of calories surpasses the maximum calories allowed for the patient

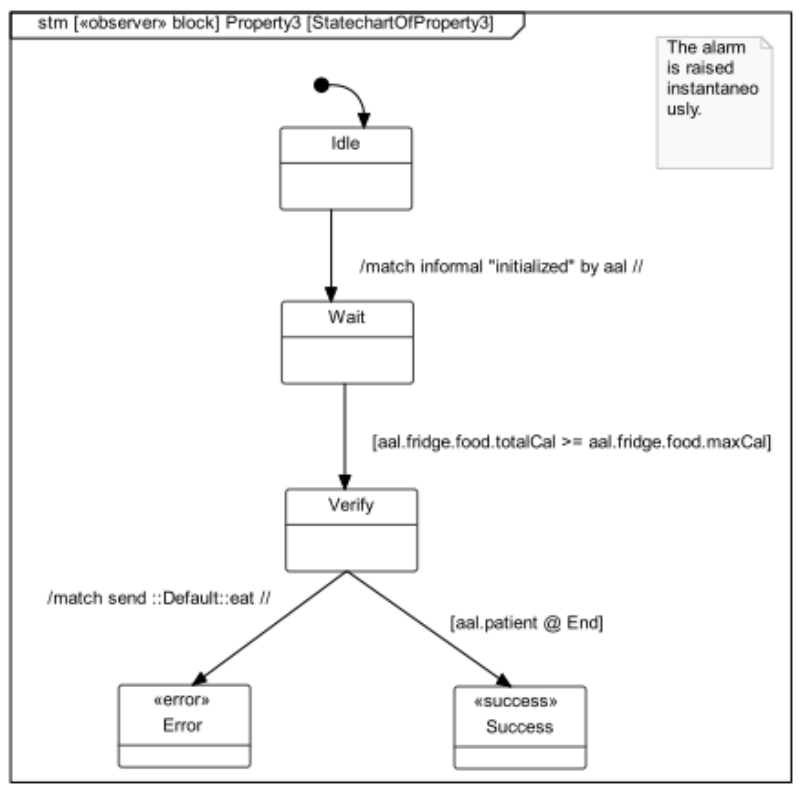

Figure 6: Property2 State Machine Diagram

This property ensures that the Patient should stop eating when the total number of calories surpasses the maximum calories allowed and that the Alarm should be raised. Fig. 6 shows the state machine diagram of the property 2 . This requirement implies that the Alarm shall be immediately raised as soon as the total number of calories equals or surpasses the maximum calories allowed for the Patient. If it happens then the Patient should stop eating. 


\section{Verification Results}

Until now, we have modeled the AAL system and the properties to be verified on the model. It is now time to verify these properties and in case if there is error during its verification, simulate it to find the error and then correct it in the model. Fig. 7 shows the snapshot of the compilation of the AAL model named AAL2. The AAL2 model is first exported into AAL2.xmi and then using the IFx toolset the AAL2.xmi is compiled into AAL2.if.

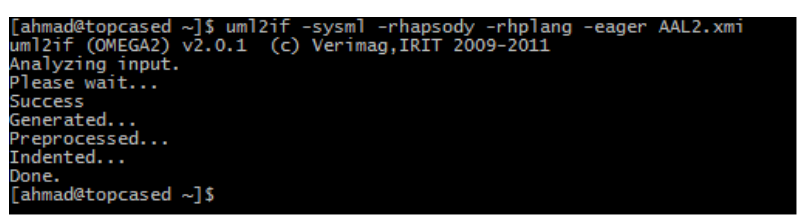

Figure 7: XMI to IF Compilation

The AAL2.if is then compiled into an executable file i.e. AAL2.x. For the properties verification part, we run the model-checker with the following options: AAL2. $\mathrm{x}-\mathrm{dfs}$-po -me -ce ln

While verifying the AAL model, the modelchecker has found several error scenarios. Any of the error scenario can then be loaded through the interactive simulation interface of the IFx toolset to trace back the error in the model and then correct it. In order to debug a model, firstly we im-

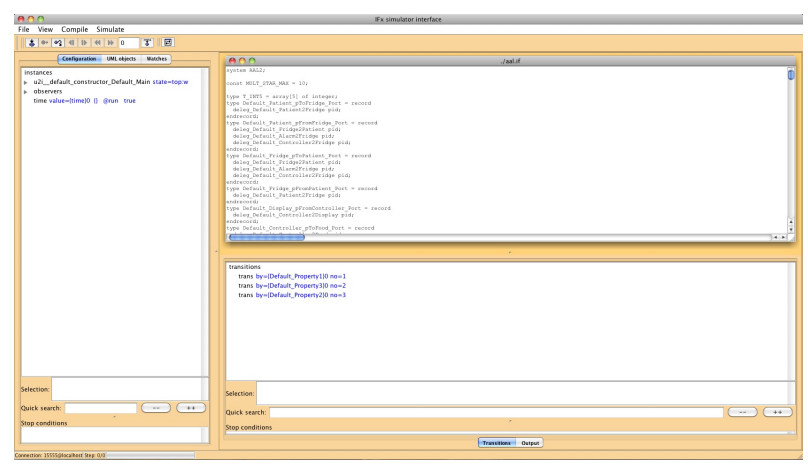

Figure 8: Initial Simulation Interface

port it into the simulator as shown in Fig. 8. We check the states of the observers in order to identify which property has not been satisfied. One can observe in Fig. 9 that Property 2 fails. While checking the state of the entire system for this property, we discover that the error state contained the maximum allowed number of calories for the total number of calories consumed and subsequently eat requests sent by the Patient.
This implies that the Alarm function of the intelligent Fridge doesn't function properly. The Alarm function of the Fridge is strictly linked to its Food process and the Alarm is raised only if the total number of consumed calories is strictly superior than the maximum allowed; condition which doesn't satisfy the request that the Alarm is raised as soon as possible. The correction consists in raising the Alarm in case the total number of consumed calories is equal to the maximum allowed threshold. Once this error is corrected the verification succeeds.

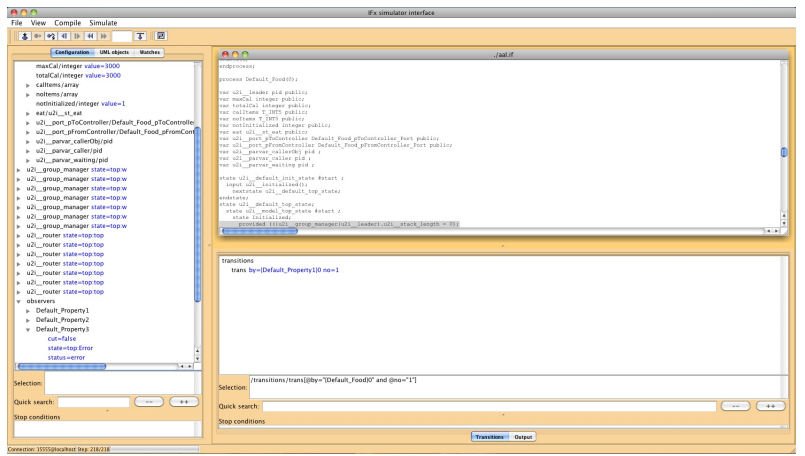

Figure 9: Error State Food Observer Simulation Interface

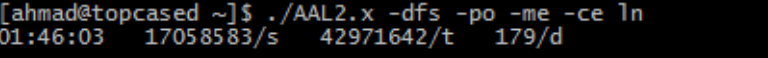

Figure 10: Model checking successful

Fig. 10 shows the result of the model-checker on the correct model.

\section{Conclusion}

We have modeled the structural and behavioral parts of an AAL (Ambient Assisted Living) system. The modeling is done using Rational Rhapsody 7.5.2 with OMEGA2 profile which is used for specification and verification of dynamic properties of models through observers. For the verification and simulation part, we have used IFx which is a toolset used for the simulation of OMEGA2 models and the verification of properties defined on these models. We have verified two properties of the AAL system using the IFx toolset. At first, the verification results in errors which can then be simulated through the interactive simulation interface of the IFx toolset in order to identify the source of the error and then subsequently correct it in the model, after correcting the error in the 
model, the verification results in the fulfillment of all the two properties.

The future work is centered around the use of formal methods in the context of our integrated approach [18].

In [7], we have investigated the use of goal oriented concepts in combination with RELAX for modeling the requirements of ambient systems. We have found a link between RELAX and SysML/KAOS [8], which is a goal oriented approach based on KAOs and which extends the SysML meta-model with goal concepts. Based on that, we have concluded that these two approaches are complementary with each other and RELAX can benefit from the ContributionNature and ContributionType concepts of SysML/KAOS.

This work motivated us to take benefit from the OMEGA2/IFx. In our integrated approach, we are interested in RELAX requirement which we obtain by using the RELAX process. We then refine the RELAX requirement with the ContributionType and ContributionNature of SysML/KaOs which is a SYSML profile with the goal concepts integrated into it, the reader is referred to [7] for an insight on our work on RELAX and SysmL/Kaos. The Relax requirement is then verified by a test case and then the test case can be refined by observer and then observer is allocated to a state machine diagram. This whole sequence of steps constitute our process.

\section{REFERENCES}

[1] http://www-2.cs.cmu.edu/ svc/

[2] Iulia Dragomir, Iulian Ober and David Lesens. A Case Study in Formal System Engineering with SysML, ICECCS 2012,Pages: $189-198$.

[3] Iulian Ober and Iulia Dragomir. OMEGA2: A new version of the profile and the tools, ICECCS 2010, DOI 10.1109/ICECCS.2010.59.

[4] Iulian Ober, Susanne Graf and Ileana Ober. Validating timed UML models by simulation and verification, International Journal on Software Tools for Technology (2006) 8(2): 128145

[5] E. M. Clarke, O. Grumberg, and D. A. Peled, Model Checking. MIT Press, 1999.

[6] Jon Whittle, Pete Sawyer, Nelly Bencomo, Betty H.C. Cheng, and Jean-Michel Bruel.
RELAX : Incorporating Uncertainty into the Specication of Self-Adaptive systems, RE Conference 2009, Pages: 79-88.

[7] Manzoor Ahmad, Jean Michel Bruel, Régine Laleau and Christophe Gnaho. Using RELAX, SysML and KAOS for Ambient Systems Requirements Modeling, Procedia Computer Science Volume 10, 2012, Pages 474481.

[8] Christophe Gnaho, Farida Semmak. Une extension SysML pour l'ingénierie des exigences non-fonctionnelles orientée but. Revue Ingénierie des Systèmes d'Information, Vol 16/1, 23 pages, 2011.

[9] Kawtar Benghazi, María Visitación Hurtado, María Luisa Rodríguez, Manuel Noguera. Applying Formal Verification Techniques to Ambient Assisted Living Systems, OTM 2009 Workshops, pp 381-390.

[10] Jürgen Nehmer, Martin Becker, Arthur Karshmer and Rosemarie Lamm. Living assistance systems: an ambient intelligence approach, ICSE '06, Pages 43-50.

[11] Ludovic Apvrille, Pierre de Saqui-Sannes, Ferhat Khendek. TURTLE-P: a UML profile for the formal validation of critical and distributed systems, Software and System Modeling 5(4): 449-466 (2006).

[12] http://www.it.uu.se/research/group/ darts

[13] I. Ober and I. Dragomir, "Unambiguous UML Composite Structures: The OMEGA2 Experience," in SOFSEM 2011: Theory and Practice of Computer Science, Springer, 2011, vol. 6543, pp. 418-430.

[14] S. Bornot and J. Sifakis, "An algebraic framework for urgency," Information and Computation, vol. 163, 2000.

[15] M. Bozga, S. Graf, I. Ober, I. Ober, and J. Sifakis, "The IF Toolset," in Formal Methods for the Design of Real-Time Systems, Springer, 2004, vol. 3185, pp. 131-132.

[16] OMEGA2-IFx for UML/SysML v2.0 Profile and Toolset, User Manual Document v1.1

[17] Manzoor Ahmad, Iulia Dragomir. AAL System Properties Modeling and Verification Using OMEGA2/IFx, Internal Report, Université de Toulouse.

[18] Jean-Michel Bruel, Nicolas Belloir and Manzoor Ahmad. SPAS: un profile SYSML pour les systèmes auto-adaptatifs, CNRIUT, Lille June 2009. 